\title{
Generic eigenvalue assignment by memoryless real output feedback
}

\author{
Joachim Rosenthal ${ }^{\mathrm{a}, \mathrm{l}}$, J.M. Schumacher ${ }^{\mathrm{b}, *}$, Jan C. Willems $^{\mathrm{c}}$ \\ a University of Notre Dame, Notre Dame, IN 46556, USA \\ ${ }^{\text {b } C W I, ~ P . O . ~ B o x ~ 94079, ~} 1090$ GB Amsterdam. The Netherlands, and Tilburg University, CentER and Department of Economics, \\ P.O. Box 90153, 5000 LE Tilburg, Netherlands \\ ${ }^{c}$ Mathematics Institute, University of Groningen, P.O. Box 800, 9700 AV Groningen, Netherlands
}

Received 20 January 1995; revised 25 April 1995

\begin{abstract}
By extensive use of methods from algebraic geometry, $X$. Wang proved that arbitrary pole placement by static output feedback is generically possible for strictly proper plants with $n$ states, $m$ inputs, and $p$ outputs, if $n<m p$. Here we show the same result using no more of algebraic geometry than the definition of genericity.
\end{abstract}

Keywords: Eigenvalue assignment; Pole assignment; Linear systems; Genericity

\section{Introduction}

In this paper we will prove in an elementary way that for generic real systems

$\frac{\mathrm{d}}{\mathrm{d} t} x=A x+B u, \quad y=C x$

having $m$ inputs, $p$ outputs, and $n$ states, with $n<$ $m p$, there exists, for each real monic polynomial $\phi$ of degree $n$, a memoryless real feedback law $u=K y$ such that the controlled system $(\mathrm{d} / \mathrm{d} t) x=(A+B K C) x$ has characteristic polynomial $\phi$ :

$$
\operatorname{det}(s I-A-B K C)=\phi(s) \text {. }
$$

To be precise, identify the set of monic real polynomials of degree $n$ with the vector space $\mathbb{R}^{n}$ and identify the set of compensators of size $m \times p$ with the vector space $\mathbb{R}^{m p}$. Recall that a subset $S$ of $\mathbb{R}^{k}$ is called

\footnotetext{
* Corresponding author. E-mail: Hans.Schumacher@cwi.nl.

1 This author was supported in part by NSF grant DMS-9400965 . The research for this paper was carried out while he was a visitor at $\mathrm{CWI}$.
}

a generic set if its complement $\mathbb{R}^{k} \backslash S$ is contained in the zero set of some nonzero polynomial in $x_{1}, \ldots, x_{k}$. We will prove:

Theorem 1. If $n<m p$ then the pole placement map

$$
\begin{aligned}
\chi: \quad \mathbb{R}^{m p} & \rightarrow \mathbb{R}^{n} \\
K & \mapsto \operatorname{det}(s I-A-B K C)
\end{aligned}
$$

is surjective for a generic set of real matrices $(A, B, C)$ of sizes $n \times n, n \times m$, and $p \times n$, respectively.

This result itself is not new. It was first proven by Wang in [12]. However, our proof is new and elementary.

The problem at hand is called the generic eigenvalue assignment problem by memoryless real output feedback. Clearly, if the McMillan degree $n$ exceeds the number of parameters $m p$, then by a simple dimension argument one concludes that the pole placement map $\chi$ cannot have dense image, let alone be surjective. The best condition which one can hope for is therefore $n \leqslant m p$ and Wang's theorem 
misses this best possible bound by only one degree of freedom.

The problem of pole assignment by static output feedback has a history going back 25 years, the first contribution being due to Davison [5]. In 1975, Kimura [7] showed that if the system $(A, B, C)$ is controllable and observable and if the McMillan degree $n$ does not exceed $m+p-1$, then almost all closedloop polynomials can be obtained, i.e. the image of $\chi$ is dense under those assumptions. A general differentiable nonlinear map can only have dense image if its linearization around at least one point is surjective, i.e. the Jacobian at that point has full row rank. For polynomial mappings between complex spaces, this necessary condition is actually also sufficient; that is the content of the so-called dominant morphism theorem. Since the pole placement map $\chi$ is indeed polynomial, Hermann and Martin [6] could use this theorem to establish the result that $n \leqslant m p$ is actually already a sufficient condition for the complexified map $\chi$ to be almost surjective for the generic complex system $(A, B, C)$.

Unfortunately, in the real case the linearization argument can only show that locally the map is surjective and nothing can be said about the global situation. In fact, soon after the Hermann-Martin paper, Willems and Hesselink [17] proved that arbitrary pole assignment is not generically possible for real systems with $m=p=2, n=4$.

In 1981, Brockett and Byrnes [2] showed that the problem of pole placement by static output feedback is in fact equivalent to a classical Schubert problem. In this way they were able to show that if $n=m p$ the complex pole placement map is surjective (rather than just almost surjective) for the generic system $(A, B, C)$. Using a calculation already performed by Schubert in the 19th century, they showed moreover that there are exactly

$d(m, p)=\frac{1 ! 2 ! \cdots(p-1) !(m p) !}{m !(m+1) ! \cdots(m+p-1) !}$

complex feedback matrices assigning a given closedloop polynomial. Since the solution set has to be closed under complex conjugation when the matrices are real and since $d(m, p)$ is odd whenever $\min (m, p)=1$ or $\min (m, p)=2$ and $\max (m, p)=2^{k}-1$, Brockett and Bymes thus showed that in these situations $n \leqslant m p$ is also a sufficient condition over the reals. The paper of Brockett and Byrnes was significant in a different respect as well since they showed that generalized compensators of the form $K_{1} y+K_{2} u=0$ (with $K_{1}$ not necessarily invertible) should be considered.

During the eighties there were numerous papers which showed that Kimura's bound could be improved, and we refer to $[3,9,11]$ and in particular to the survey article of Byrnes [4] where also more complete references can be found.

In 1992, Wang [12], using techniques from algebraic geometry, was first able to prove Theorem 1. Only later it was independently recognized by Leventides $[8,10]$, Wang [13] and Ariki [1] that the geometric techniques used by Wang in [12] are actually based on a linearization of the pole placement map around a so-called 'dependent' compensator. All these papers use polynomial representations. One of the advantages of this approach is that it is easy to construct a dependent compensator (see e.g. [12]). On the other hand, in order to use the concept of genericity one needs to equip the set of polynomial matrices of a fixed McMillan degree with the structure of an algebraic variety, which can be done but is a nontrivial task: one either has to work in a so-called Quot scheme, or alternatively one uses the connection with state space representations but then one essentially has to show that 'realization theory is algebraic'.

In this paper we will stay strictly in the state space framework. Not only does this mean that we now solve the problem in the context in which it was originally stated, but we also avoid the difficulties associated with the translation from the state space framework to the polynomial setting. Our basic tool however is still the linearization around a dependent compensator, and we provide in Lemma 6 a closed formula for the Jacobian in terms of the matrices $A, B$, and $C$.

\section{Preliminaries}

In this section we will summarize the mathematical preliminaries that we will need in this paper. Consider a linear system of the form (1) where $A, B$, and $C$ are matrices of sizes $n \times n, n \times m$, and $p \times n$, respectively. We shall consider static compensators of the general form

$K_{1} y+K_{2} u=0$

where $\left[\begin{array}{ll}K_{1} & K_{2}\end{array}\right]$ is an $m \times(p+m)$ matrix of full row rank. Interconnection of (1) and (4) results in the controlled behavior. See $[15,16]$ for more details about the behavioral approach and feedback interconnections in this context. 
The subspace $\operatorname{ker}\left[\begin{array}{ll}K_{1} & K_{2}\end{array}\right]$ can of course also be given in image representation $\operatorname{im}\left[\begin{array}{l}F_{1} \\ F_{2}\end{array}\right]$, where $F:=$ $\left[\begin{array}{l}F_{1} \\ F_{2}\end{array}\right]$ is a $(p+m) \times p$ matrix of full column rank. The behavior of the compensator (4) can then be described as well by

$y=F_{1} \ell, \quad u=F_{2} \ell$,

where $\ell$ is a latent variable, and the combined behavior of the system and the compensator is then described through

$$
\left[\begin{array}{cc}
\frac{\mathrm{d}}{\mathrm{d} t} I-A & -B F_{2} \\
-C & F_{1}
\end{array}\right]\left[\begin{array}{l}
x \\
\ell
\end{array}\right]=0 .
$$

We define the unnormalized closed-loop characteristic polynomial by

$\phi_{F}(s):=\operatorname{det}\left[\begin{array}{cc}s I-A & -B F_{2} \\ -C & F_{1}\end{array}\right]$.

If the matrix $F_{1}$ is invertible, then the feedback law (5) can also be written $u=F_{2} F_{1}^{-1} y$. In this case the polynomial $\phi_{F}(s)$ always has degree $n$, and the standard (normalized) closed-loop characteristic polynomial corresponding to $K=F_{2} F_{1}^{-1}$, as defined in (2), is obtained from it by dividing through by the leading coefficient of $\phi_{F}(s)$. Actually the invertibility of $F_{1}$ is not only sufficient but also necessary for the unnormalized closed-loop polynomial to have degree $n$; this follows from Laplace's expansion of the determinant in terms of minors of order $p$, or also from (10) below. The compensator given by (5) is said to be nonproper if $F_{1}$ is not invertible (equivalently, if $\left.\operatorname{deg} \phi_{F}(s)<n\right)$, and dependent if $\phi_{F}(s)=0$. Dependent compensators will play a central role in the development below.

It will be convenient to use the following alternative form of the unnormalized closed-loop characteristic polynomial.

Lemma 2. Let a system $(A, B, C)$ be given, and write $G(s):=C(s I-A)^{-1} B$. Then the unnormalized closed-loop characteristic polynomial corresponding to the compensator (5) satisfies

$\phi_{F}(s)=\operatorname{det}(s I-A) \operatorname{det}\left(\left[\begin{array}{ll}I_{p} & -G(s)\end{array}\right] F\right)$.

Proof. By the well-known 'Schur complement formula', which states that

$\operatorname{det}\left[\begin{array}{ll}M_{11} & M_{12} \\ M_{21} & M_{22}\end{array}\right]=\operatorname{det} M_{11} \operatorname{det}\left(M_{22}-M_{21} M_{11}^{-1} M_{12}\right)$ whenever $M_{11}$ is invertible, we get

$$
\phi_{F}(s)=\operatorname{det}(s I-A) \operatorname{det}\left(F_{1}-C(s I-A)^{-1} B F_{2}\right)
$$

which is the same as the formula in the statement of the lemma.

From (9), one easily determines the coefficient of $s^{n}$ in the unnormalized closed-loop characteristic polynomial:

$\phi_{F}(s)=\left(\operatorname{det} F_{1}\right) s^{n}+$ lower-order terms in $s$.

The map that takes a matrix $F \in \mathbb{P}^{(m+p) \times p}$ to the corresponding unnormalized closed-loop characteristic polynomial $\phi_{F}(s)$ will be called the extended pole placement map, and we shall denote it by $\hat{\chi}$. Identifying the space of matrices $\mathbb{R}^{(m+p) \times p}$ with the linear space $\mathbb{R}^{(m+p) p}$, we can in fact consider $\hat{\chi}$ as a mapping defined on all of $\mathbb{R}^{(m+p) p}$ since the expression at the right-hand side in (7) is also well-defined if $F$ does not have full column rank. If we also identify the space of all polynomials of degree at most $n$ with $\mathbb{R}^{n+1}$, then we can think of $\hat{\chi}$ simply as a mapping between two linear spaces:

$$
\begin{aligned}
\hat{\chi}: \quad \mathbb{R}^{(p+m) p} & \rightarrow \mathbb{R}^{n+1} \\
F & \mapsto \phi_{F}(s) .
\end{aligned}
$$

Of course, $\hat{\chi}$ is a nonlinear map, in fact a polynomial one. The following properties of $\hat{\chi}$ are immediate from the definition (7), or also from Lemma 2:

(i) $\hat{\chi}(F S)=\hat{\chi}(F)$ for any matrix $S \in \mathbb{R}^{p \times p}$ that has determinant 1 ;

(ii) $\hat{\chi}(\lambda F)=\lambda^{p} \hat{\chi}(F)$ for $\lambda \in \mathbb{R}$.

At each point of $F \in \mathbb{R}^{(p+m) p}$, one may compute the Jacobian of $\hat{\chi}$, denoted by $\mathrm{d} \hat{\chi}(F)$. The Jacobian is a matrix of size $(n+1) \times(p+m) p$, and by the standard multivariable Taylor series expansion one has

$$
\begin{aligned}
\hat{\chi}(F+\varepsilon H)= & \hat{\chi}(F)+\varepsilon \mathbf{d} \hat{\chi}(F) H \\
& + \text { terms of higher order in } \varepsilon
\end{aligned}
$$

for $H$ in $\mathbb{R}^{(p+m) p}$. As a consequence of property (i) above, the rank of the Jacobian can be at most $(p+$ $m) p-\left(p^{2}-1\right)=m p+1$. Compensators for which the Jacobian $\mathrm{d} \hat{\chi}(F)$ has full row rank will be important in the sequel, and therefore we introduce the following terminology.

Definition 3. Consider a linear system of the form (1) and a memoryless compensator given by $F=\left[\begin{array}{l}F_{1} \\ F_{2}\end{array}\right] \in$ $\mathbb{R}^{(p+m) p}$ as in (5). The compensator will be called full 
if the Jacobian of the extended pole placement map at $F, \mathrm{~d} \hat{\chi}(F)$, has full row rank.

Remark 4. The matrix $\mathrm{d} \hat{\chi}(F)$ will not have full row rank if and only if all minors of size $(n+1) \times(n+1)$ vanish. These minors are polynomial functions of the entries of $\mathrm{d} \hat{\chi}(F)$. Because $\hat{\chi}$ depends polynomially on the entries of $F$, the entries of the Jacobian in their turn also depend polynomially on the entries of $F$. We conclude therefore that the collection of matrices $F$ corresponding to compensators that are not full is described by a set of polynomial equations. These equations may be always fulfilled, however. In fact, it follows from the remarks above that the set of full compensators $F$ must be empty if $n>m p$.

We recall here the definition of the adjoint of a matrix (cf. for instance [14, p. 7]), which will be needed at several places below. Let $M$ be an $n \times n$ matrix. The adjoint of $M$, denoted by adj $M$, is the $n \times n$ matrix defined by

$(\operatorname{adj} M)_{i j}:=\left((-1)^{i+j} M_{j i}\right)_{i, j=1}^{n}$

where $M_{j i}$ denotes the determinant of the $(n-1) \times$ $(n-1)$ matrix obtained from $M$ by removing the $j$ th row and the $i$ th column. By the properties of determinants, one has

$\sum_{j} m_{i j}(\operatorname{adj} M)_{j k}= \begin{cases}\operatorname{det} M & \text { if } i=k, \\ 0 & \text { otherwise }\end{cases}$

Another way to write this is

$M \cdot \operatorname{adj}(M)=\operatorname{det} M \cdot I_{n}$,

from which one sees that the adjoint is closely related to the inverse. Another consequence of (14) is that, if $\operatorname{det} M$ is viewed as a function of the matrix elements $m_{i j}$, one has

$\frac{\partial}{\partial m_{i j}} \operatorname{det} M=(\operatorname{adj} M)_{j i}$.

If the elements $m_{i j}$ are differentiable functions of some parameter $\varepsilon$, we therefore have, by the chain rule,

$\frac{\mathrm{d} M}{\mathrm{~d} \varepsilon}(\varepsilon)=\sum_{j} \sum_{i}(\operatorname{adj} M(\varepsilon))_{j i} m_{i j}^{\prime}(\varepsilon)$.

Writing the right-hand side in a different way, we get the formula

$\frac{\mathrm{d} M}{\mathrm{~d} \varepsilon}(\varepsilon)=\operatorname{tr}\left[(\operatorname{adj} M(\varepsilon)) M^{\prime}(\varepsilon)\right]$ where of course 'tr' denotes trace (the sum of the diagonal elements).

\section{The linearization around a dependent compensator}

We now establish a sufficient criterion (Lemma 5) which guarantees that the extended pole placement map $\hat{\chi}$ is surjective over the reals. The criterion is based on a linearization around a dependent compensator, an idea which appeared first in purely geometric language in [12] and was later worked out in more elementary terms independently by Leventides $[8,10]$, Wang [13] and Ariki [1].

Lemma 5. If a dependent full compensator exists for a system $(A, B, C)$, then the extended pole placement map $\hat{\chi}$ given by (11) is surjective. Consequently, in this case the system $(A, B, C)$ allows arbitrary pole placement by static output feedback.

Proof. Let $F$ be dependent and full so that $\mathrm{d} \hat{\chi}(F)$ is surjective. By the inverse function theorem, an open neighborhood of $F$ is mapped onto an open neighborhood, say $V$, of $\hat{\chi}(F)=0$. Because any polynomial $\phi$ of degree at most $n$ may be written as $\phi=c \phi_{0}$ with $\phi_{0} \in V$ for some (sufficiently large) $c \in \mathbb{R}$, and since $\hat{\chi}(\hat{\lambda} F)=\lambda^{p} \hat{\chi}(F)$ for any $\hat{\lambda} \in \mathbb{R}$, this is already enough to prove that $\hat{\chi}$ is surjective. But then also the regular pole placement map $\chi$ defined by ( 3 ) is surjective. Indeed, let a polynomial $\phi(s)$ of degree $n$ with leading coefficient 1 be given, and let $F=\left[\begin{array}{l}F_{1} \\ F_{2}\end{array}\right]$ be such that $\hat{\chi}(F)=\phi$. Then $F_{1}$ must be invertible by $(10)$, and we have $\phi=\chi(K)$ for $K=F_{2} F_{1}^{-1}$.

To the extent that the inverse function theorem is constructive, the proof provides also a way to compute a compensator that will assign a given set of poles. This is shown in detail by Wang [13].

Since the closed-loop characteristic polynomial $\phi_{F}(s)$ is a polynomial of degree at most $n$, we may define functions $a_{r}(F)(0 \leqslant r \leqslant n)$ by

$$
\begin{aligned}
\phi_{F}(s)= & a_{n}(F) s^{n}+a_{n-1}(F) s^{n-1} \\
& +\cdots+a_{1}(F) s+a_{0}(F) .
\end{aligned}
$$

The $a_{r}(F)$ depend polynomially on the $n^{2}+$ $(n+p)(m+p)$ entries of the matrices $A, B, C$, and $F$; in particular they are differentiable. For a given 
compensator $F$, we can therefore define a $(p+m) p$ tuple of polynomials $\phi_{F}^{i j}(s)$ given by

$$
\begin{aligned}
\phi_{F}^{i j}(s)= & \frac{\partial a_{n}}{\partial f_{i j}}(F) s^{n}+\cdots+\frac{\partial a_{1}}{\partial f_{i j}}(F) s \\
& +\frac{\partial a_{0}}{\partial f_{i j}}(F) .
\end{aligned}
$$

These polynomials indicate the directions into which the closed-loop characteristic polynomial can be moved by slightly perturbing the elements of $F$. In other words, the coefficients of the polynomials $\phi_{F}^{i j}(s)$ are the columns of the Jacobian matrix $\mathrm{d} \hat{\chi}(F)$. One way to verify that a given compensator is full therefore is to check whether these polynomials together span the $(n+1)$-dimensional space of all polynomials of degree at most $n$. The following lemma shows a convenient way to compute the polynomials $\phi_{F}^{i j}(s)$.

Lemma 6. The Jacobian $\mathrm{d} \hat{\chi}(F)$ of the extended pole placement map $\hat{\chi}$ at the compensator $F$ is given by

$$
\begin{aligned}
\mathrm{d} \hat{\chi}(F): H \mapsto \operatorname{det}(s I-A) \operatorname{tr}\left(\operatorname{adj}\left(\left[I_{p}-G(s)\right] F\right)\right. \\
\left.\times\left[I_{p}-G(s)\right] H\right) .
\end{aligned}
$$

In particular, the polynomials $\phi_{F}^{i j}(s)$ defined by (20) are equal to the entries of the matrix

$$
\operatorname{det}(s I-A)\left(\operatorname{adj}\left[F_{1}-G(s) F_{2}\right]\right)\left[I_{p}-G(s)\right] .
$$

Proof. Take $H \in \mathbb{R}^{(p+m) p}$. According to Lemma 2 and formula (18), we have

$$
\begin{aligned}
& \mathrm{d} \hat{\chi}(F) H \\
& \begin{array}{l}
=\left.\frac{\mathrm{d}}{\mathrm{d} \varepsilon}\left(\operatorname{det}(s I-A) \operatorname{det}\left(\left[I_{p}-G(s)\right](F+\varepsilon H)\right)\right)\right|_{\varepsilon=0} \\
=\operatorname{det}(s I-A) \frac{\mathrm{d}}{\mathrm{d} \varepsilon}\left(\operatorname { d e t } \left(\left[I_{p}-G(s)\right] F\right.\right. \\
\left.\left.\quad+\varepsilon\left[I_{p}-G(s)\right] H\right)\right)\left.\right|_{\varepsilon=0}
\end{array} \\
& =\operatorname{det}(s I-A) \operatorname{tr}\left(\operatorname{adj}\left(\left[I_{p}-G(s)\right] F\right)\left[I_{p}-G(s)\right] H\right) .
\end{aligned}
$$

The polynomials $\phi_{F}^{i j}(s)$ are obtained from this formula by setting $H=E_{i j}$, where $E_{i j}$ denotes the $(p+m) \times p$ matrix, all of whose entries are zero except for the one in the $(i, j)$ position, which has value 1 . Since $\operatorname{tr}\left(M E_{i j}\right)=m_{j i}$ for any $p \times(p+m)$ matrix $M$ with entries $m_{i j}$, one obtains in this way indeed all the entries of the matrix in (22).

\section{An elementary proof of Wang's condition for pole placement by static output feedback}

In this section we will provide the proof of Theorem 1. Let us first explain the strategy of the proof. Because of Lemma 5, it suffices to show that for the generic plant with $m$ inputs, $p$ outputs, and $n<$ $m p$ states, it is possible to construct a dependent full compensator. While it is known that dependent compensators generically do not exist if $n \geqslant m p$, it is quite trivial to show that such compensators can always be found if $n<m p$ (compare [3] and [12, Proposition 4.1]). However, in order to accomplish our proof we shall in fact need a procedure to construct a dependent compensator that is expressed polynomially in the entries of the given matrices $(A, B, C)$. For this particular compensator, the fullness condition needed in Lemma 5 is then expressed through polynomial conditions in the entries of $(A, B, C)$ (see Remark 4). It follows that the collection of triples $(A, B, C)$ that do not allow a dependent full compensator is contained in a set that is described by polynomial equations. To show that arbitrary pole assignment is possible generically, it suffices to show that these polynomial equations are nontrivial. For this it is enough to produce an example of a triple $(A, B, C)$ for which the dependent compensator constructed by the polynomial procedure is indeed full. In the two subsections below we shall first give the polynomial construction of a dependent compensator, and then provide the example that is needed to prove genericity.

\subsection{Polynomial construction of a dependent compensator}

We start from the following data: an $n \times n$ matrix $A$, an $n \times m$ matrix $B$, and a $p \times n$ matrix $C$, with $n<m p$. In this subsection we construct a $(p+m) \times p$ matrix $F$ whose entries are polynomial functions of the entries of $A, B$, and $C$, and which is such that $\phi_{F}=0$.

As is seen from (7), the dependency condition comes down to verifying whether a matrix of the form $s M-N$ has determinant zero. For this one has the following well-known criterion; we provide a short proof for completeness.

Lemma 7. Let $M$ and $N$ be square matrices. We have $\operatorname{det}(s M-N)=0$ if and only if there exist vectors $z_{0}, \ldots, z_{k}$ such that $M z_{0}=0, M z_{i+1}=N z_{i}$ for $i=$ $0, \ldots, k-1$, and $M z_{k}=0$. 
Proof. For a general square rational matrix $T(s)$, we have that $\operatorname{det} T(s)=0$ if and only if there exists a polynomial vector $z(s)$ such that $T(s) z(s)=0$. In the special case $T(s)=s M-N$, this condition may be reformulated as in the statement of the lemma by writing $z(s)=z_{0} s^{k-1}+z_{1} s^{k-2}+\cdots+z_{k-1}$.

The polynomial formula for a dependent compensator will be based on the following lemma.

Lemma 8. Let $A, B$, and $C$ be matrices of sizes $n \times n$, $n \times m$, and $p \times n$, respectively, and suppose there exist m-vectors $u_{0}, \ldots, u_{k-1}$ such that

$A^{k-1} B u_{0}+\cdots+A B u_{k-2}+B u_{k-1}=0$.

Define p-vectors $y_{0}, \ldots, y_{k-1}$ by

$$
\begin{aligned}
x_{0} & =0, \quad x_{i+1}=A x_{i}+B u_{i}, \\
y_{i} & =C x_{i} \quad(i=0, \ldots, k-1) .
\end{aligned}
$$

If $F$ is any full column rank matrix of size $(p+m) \times$ $p$ whose column span contains all the vectors $\left[\begin{array}{l}y_{i} \\ u_{i}\end{array}\right]$ $(i=0, \ldots, k-1)$, then the compensator given by $F$ is dependent.

Proof. Let the conditions of the lemma be satisfied. By assumption, there exist $p$-vectors $\ell_{0}, \ldots, \ell_{k-1}$ such that

$\left[\begin{array}{l}y_{i} \\ u_{i}\end{array}\right]=\left[\begin{array}{l}F_{1} \\ F_{2}\end{array}\right] \ell_{i} \quad(i=0, \ldots, k-1)$.

Define $\ell_{k}=0$. One verifies that the criterion of Lemma 7 holds with

$M=\left[\begin{array}{ll}I & 0 \\ 0 & 0\end{array}\right], \quad N=\left[\begin{array}{cc}A & B F_{2} \\ C & -F_{1}\end{array}\right], \quad z_{i}=\left[\begin{array}{c}x_{i} \\ \ell_{i}\end{array}\right]$

where $x_{i}$ is defined as in (24). In particular, note that $M z_{k}=0$ by (23). By Lemma 2, this means that the compensator given by $F$ is dependent.

We now come to the promised polynomial construction of a dependent compensator. In this construction we assume that $n<m p$. Write $n$ as a multiple of $m$ plus a remainder, so

$n=(k-1) m+r, \quad 0 \leqslant r<m$,

where we have $0<k \leqslant p$ by the assumption $n<m p$. The controllability matrix $\left[\begin{array}{lllll}B & A B & \cdots & A^{k-1} B\end{array}\right]$ has $n$ rows and $k m>n$ columns, so it is a 'strictly flat' matrix. We extend it to a square matrix of size $\mathrm{km} \times \mathrm{km}$ by adding a number of standard unit row vectors:

$Q \stackrel{\text { def }}{=}\left[\begin{array}{cccc}B & A B & \cdots & A^{k-1} B \\ O & & I_{k m-n}\end{array}\right]$.

Now, define a sequence $u_{0}, \ldots, u_{k-1}$ by

$$
\begin{aligned}
& {\left[\begin{array}{llll}
u_{k-1}^{\mathrm{T}} & u_{k-2}^{\mathrm{T}} & \cdots & u_{0}^{\mathrm{T}}
\end{array}\right]^{\mathrm{T}}} \\
& \quad=\text { rightmost column of adj } Q .
\end{aligned}
$$

Note that the vectors $u_{i}$ depend polynomially on the entries of $A$ and $B$; by (15) and because $\mathrm{km}$ is strictly less than $n$, they also satisfy (23). Next define $y_{0}, \ldots, y_{k-1}$ as in (24). Finally, define

$F=\left[\begin{array}{ll}F_{11} & F_{12} \\ F_{21} & F_{22}\end{array}\right]$

with

$$
\begin{aligned}
& F_{11}=\left[\begin{array}{lll}
y_{0} & \cdots & y_{k-1}
\end{array}\right], F_{12}=\left[\begin{array}{c}
0 \\
I_{p-k}
\end{array}\right], \\
& F_{21}=\left[\begin{array}{lll}
u_{0} & \cdots & u_{k-1}
\end{array}\right], F_{22}=0 .
\end{aligned}
$$

The matrix $F$ depends polynomially on the entries of $A, B$, and $C$, and by Lemma 8 , we have $\phi_{F}=0$. In the next subsection we shall show that this $F$ generically determines a compensator that is not only dependent but also full in the sense of Definition 3.

\subsection{Proof of genericity}

We know from Lemma 5 that arbitrary pole placement is possible for a system $(A, B, C)$ if a matrix $F$ can be found such that $\hat{\chi}(F)=0$ and $\mathrm{d} \hat{\chi}(F)$ has full row rank. Above we constructed, under the assumption $m p<n$, an $F=F(A, B, C)$ depending polynomially on $(A, B, C)$ such that $\hat{\chi}(F(A, B, C))=0$. It follows that the set of triples $(A, B, C) \in \mathbb{R}^{n^{2}+m n+p n}$ for which arbitrary pole placement is not possible is contained in the set of triples $(A, B, C)$ for which all $(n+1) \times(n+1)$ minors in the matrix $\mathrm{d} \hat{\chi}(F(A, B, C))$ vanish, which is a set determined by polynomial equations. This still does not prove genericity of arbitrary pole placement however, since it might happen that the polynomial equations turn out to be trivial (of the form $0=0)$, in which case $\mathrm{d} \hat{\chi}(F(A, B, C))$ would be rank deficient for all triples $(A, B, C)$. To show that this situation does not occur, the last step in the proof therefore is to come up with one triple $(A, B, C)$ for which the Jacobian $\mathrm{d} \hat{\chi}(F(A, B, C))$ does indeed have full row rank. 
It may seem an easy task to produce an example of a system having a generic property, since 'almost anything' will do. However, we are looking for an example in which the calculations needed to verify that the property holds are not too involved, and unfortunately the very simple examples (such as $A=0, B=0, C=0$ ) belong to the exceptional set; to get arbitrary pole placement, we need at least controllability and observability. We believe that the example below comes reasonably close to being as simple as possible. Actually it is somewhat easier to produce an example (and also to do the polynomial construction of a dependent compensator) if one starts from kernel ('AR') representations [15] and for this compare with $[12,10]$. For reasons explained in the introduction we choose here to stay within the $(A, B, C)$ framework.

For typographical reasons, it is convenient to introduce some extra notation. Let $N_{r}$ be the nilpotent matrix of size $r \times r$ given by

$N_{r}:=\left[\begin{array}{cccc}0 & 1 & & 0 \\ 0 & 0 & \ddots & \\ \vdots & & \ddots & 1 \\ 0 & \cdots & \cdots & 0\end{array}\right]$

Let $e_{r} \in \mathbb{R}^{n}$ denote the $r$ th standard unit vector in the column space of $n$-vectors. Define $k$ and $r$ as in (25), and write

$$
\begin{aligned}
& \alpha_{1}=k, \quad \alpha_{2}=2 k, \ldots, \alpha_{r}=r k, \\
& \alpha_{r+1}=r k+k-1, \ldots, \alpha_{m}=r k+(m-r)(k-1) .
\end{aligned}
$$

Note that $\alpha_{m}=n$ by (25), and that $\alpha_{m}-\alpha_{m-1}=k-1$ because $r<m$.

Now, define matrices $A, B$, and $C$ as follows:

$$
\begin{aligned}
& A:=\left[\begin{array}{cc}
N_{n-k+1} & 0 \\
0 & N_{k-1}
\end{array}\right], \quad B:=\left[\begin{array}{lll}
e_{x_{1}} & \cdots & e_{\alpha_{m}}
\end{array}\right], \\
& C:=\left[\begin{array}{llllllll}
e_{1} & e_{n} & e_{n-1} & \cdots & e_{n-k+2} & 0 & \cdots & 0
\end{array}\right]^{\mathrm{T}} .
\end{aligned}
$$

The last $p-k$ rows of the matrix $C$ are zero, so the system above effectively has $k$ outputs, where $k$ may be less than $p$. We can take such a $C$ matrix because $k$ still satisfies $m k>n$.

We first have to compute the compensator produced by the polynomial construction of the previous subsection. Our choice of system parameters has been such that the matrix appear- ing in (27) is invertible, and in fact its determinant is equal to plus or minus 1 . Also, note that $A^{k-1} b_{m}=0$ where $b_{m}$ denotes the last column of the matrix $B$. Using (15), one sees that, up to a sign which has no essential effect, the vectors $u_{i}$ defined by (27) in the present case become

$u_{0}=\left[\begin{array}{llll}0 & \cdots & 0 & 1\end{array}\right]^{\mathrm{T}}, \quad u_{1}=0, \quad \ldots, \quad u_{k-1}=0$.

Following the recipe of the subsection above, one obtains

$$
F_{1}=\left[\begin{array}{cc}
0 & 0 \\
0 & I_{p-1}
\end{array}\right], \quad F_{2}=\left[\begin{array}{cccc}
0 & 0 & \cdots & 0 \\
\vdots & & & \vdots \\
0 & & & \vdots \\
1 & 0 & \cdots & 0
\end{array}\right] .
$$

Now we have to verify whether the so constructed compensator is full. For this we use the criterion given in Lemma 6. To apply the lemma, we need to compute the transfer matrix of the system (29). A limited amount of computation leads to

$$
\begin{aligned}
G(s) & =C(s I-A)^{-1} B \\
& =\left[\begin{array}{ccccc}
s^{-\alpha_{1}} & s^{-x_{2}} & \cdots & s^{-\alpha_{m-1}} & 0 \\
0 & \cdots & \cdots & 0 & s^{-1} \\
\vdots & & & \vdots & \vdots \\
0 & \cdots & \cdots & 0 & s^{-(k-1)}
\end{array}\right] .
\end{aligned}
$$

Next we compute

$$
\begin{gathered}
F_{1}-G(s) F_{2} \\
=\left[\begin{array}{ccccc}
0 & 0 & \cdots & 0 & 0 \\
-s^{-1} & 1 & \cdots & 0 & 0 \\
\vdots & & \ddots & & \\
-s^{-(k-1)} & 0 & \cdots & 1 & 0 \\
& O & & & I_{p-k}
\end{array}\right]
\end{gathered}
$$

The adjoint of this matrix is perhaps most easily obtained by noting that the adjoint of any $n \times n$ matrix of the form $\left[{ }_{T}^{0}\right]$ where $T$ has $n-1$ rows is a matrix of the form $\left[\begin{array}{ll}z & 0\end{array}\right]$, where $z$ is a vector such that $T z=0$. We get

$$
\operatorname{adj}\left(F_{1}-G(s) F_{2}\right)=\left[\begin{array}{cccc}
1 & 0 & \cdots & 0 \\
s^{-1} & 0 & \cdots & 0 \\
\vdots & \vdots & & \vdots \\
s^{-(k-1)} & 0 & \cdots & 0
\end{array}\right]
$$


Finally we compute the matrix appearing in Lemma 6:

$$
\begin{aligned}
& \operatorname{det}(s I-A) \cdot\left(\operatorname{adj}\left[F_{1}-G(s) F_{2}\right]\right)\left[I_{p}-G(s)\right]
\end{aligned}
$$

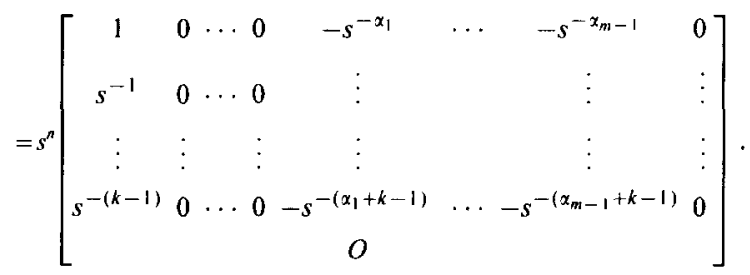

All monomials $s^{r}$ with $0 \leqslant r \leqslant n$ appear in this matrix and so the compensator we have constructed is full. With this we have shown that the generic system $(A, B, C)$ with $m p>n$ allows a dependent full compensator and therefore, according to Lemma 5 , can be arbitrarily pole assigned. This completes the proof of Theorem 1.

\section{References}

[1] S. Ariki, Generic pole assignment via dynamic feedback, Preprint, October 1994.

[2] R.W. Brockett and C.I. Bymes, Multivariable Nyquist criteria, root loci and pole placement: a geometric viewpoint, IEEE Trans. Automat. Control AC-26 (1981) 271-284

[3] C.I. Bymes, On the stability of multivariable systems and the Ljusternik-Snirelmann category, Systems Control Lett. 3 (1983) 255-262.

[4] C.I. Bymes, Pole assignment by output feedback, in: H. Nijmeijer and J.M. Schumacher, Eds., Three Decades of Mathematical System Theory, Lecture Notes in Control and
Information Sciences, Vol. 135 (Springer, Berlin, 1989) 31-78.

[5] E. Davison, On pole assignment in linear systems with incomplete state feedback, IEEE Trans. Automat. Control AC-15 (1970) 348-351.

[6] R. Hermann and C.F. Martin, Applications of algebraic geometry to system theory part I, IEEE Trans. Automat. Control AC-22 (1977) 19-25.

[7] H. Kimura, Pole assignment by gain output feedback, IEEE Trans. Automat. Control AC-20 (1975) 509-516.

[8] J. Leventides, Algebrogeometric and topological methods in control theory, Ph.D. Thesis, City University London, September 1993.

[9] J. Leventides and N. Karcanias, A new sufficient condition for arbitrary pole placement by real constant output feedback, Systems Control Lett. 18 (1992) 191-200.

[10] J. Leventides and N. Karcanias, Global asymptotic linearisation of the pole placement map: a closed form solution for the constant output feedback problem, Automatica 31 (1995) 1303-1309.

[11] J. Rosenthal, New results in pole assignment by real output feedback, SIAM J. Control Optim. 30 (1992) 203-211.

[12] X. Wang, Pole placement by static output feedback, J. Math Systems Estimation Control 2 (1992) 205-218.

[13] X. Wang, Grassmannian, central projection and output feedback pole assignment of linear systems, Preprint; to appear in IEEE Trans. Automat. Control (1994).

[14] J. Wedderburn, Lectures on Matrices, Coll. Publ. XVII (Amer. Mathematical Soc., Providence, RI, 1934).

[15] J.C. Willems, Paradigms and puzzles in the theory of dynamical systems, IEEE Trans. Automat. Control AC-36 (1991) 259-294.

[16] J.C. Willems, On interconnections, control and feedback, Preprint, July 1994

[17] J.C. Willems and W.H. Hesselink, Generic properties of the pole placement problem, in: Proc. 7th IFAC Congr. (1978) $1725-1729$. 